\title{
Construction of WeChat Public Patient Service Platform in Cancer Hospital
}

\author{
Fangfang $\mathrm{Hao}^{1, \mathrm{a}}$ and Shoujin Wang ${ }^{1, \mathrm{~b}}$ \\ School of Information \& Control engineering, Shenyang Jianzhu University, Shenyang, China \\ a 534809467@qq.com, ${ }^{\mathrm{b}}$ 23917240@qq.com
}

Keywords: WeChat public patient service platform; Cancer hospital; Internet medical treatment

\begin{abstract}
In recent years, with the continuous development of the Internet, the construction of hospital informatization has achieved remarkable results in improving the mode of medical treatment, promoting refined management, improving patient satisfaction, and improving the efficiency of medical care. Mobile medicine, on the other hand, has changed the traditional medical model with the advantages of convenience and efficiency, promoted the improvement of health services, changed medical habits, reduced the time and cost of processes such as registration, waiting for medical treatment, and receiving reports, and reduced the contradictions of doctors and patients. Make people enjoy higher quality medical experience and higher level of medical order.
\end{abstract}

\section{Introduction}

The independent appointment diagnosis and treatment through Internet means more convenient for the masses to take medical care, make full use of medical resources, maintain medical order and reduce the contradiction between doctors and patients. [1,2]It is not only a convenience to the masses, but also a more standardized working environment and work model for the medical staff[3,4]. Further use of information technology to realize the appointment diagnosis and treatment, grading diagnosis and treatment, and the new age medical model, to build a new era of digital hospital, with information as a push, to improve the level of hospital management, reduce the risk of medical security[5].

There are a large number of elderly patients in tumor hospitals, such as the large number of elderly patients, repeated hospitalization, and a large number of patients in different places[6]. Through the establishment of the WeChat public number patient service platform, our hospital further satisfies the needs of patients' appointment registration, payment, examination report, hospitalization, and payment of hospitalization advance payment[7,8]. One station solution to the patients' queuing and duplication of labor, and so on, has received the praise and support of the patients[9].

\section{The Advantages and Disadvantages of the WeChat Public Patient Service Platform}

Advantages of the WeChat public patient service platform. The whole process from outpatient registration to hospitalization, can not only save the time and energy of patients' treatment, but also improve the order of medical treatment, reduce the cost of hospital management and strengthen the safety of medical treatment. First, the patients who are registered and paid by the mobile terminal do not need to queue up to the window again. The patient's shunt is successfully realized, which greatly relieves the pressure of the window in the peak period, reduces the detained personnel in the hall, and reduces the hidden danger of security. Secondly, the patient can make an appointment to register through the mobile end time period. It can clear the time of his visit and the serial number of the doctor. It doesn't have to get up early. The patients in different places can also arrange the time reasonably, so it is not necessary to bear the embarrassment of "starting early, catching late set". Third, patients can directly inspect inspection reports and cost information through mobile terminals, and do not need to report to the past or go window to inquire about expenses. Fourth, the patient's hospitalization and payment of inpatient payment through the mobile terminal are much 
more convenient for inpatients. The patients and their relatives can pay for the bedside payment, avoid the payment of the payment of the window or delay the treatment because of the payment.

Malpractice of the WeChat public patient service platform. However, the complete process also has some drawbacks, such as the patient can not judge the Department of disease, can not be registered independently. The number of elderly patients is large, the patients unable to use WeChat also exist; when the program appears abnormal or illegal operation, the patient can not understand, and can not be handled by themselves.

Solution. For patients with larger or less access to Internet applications, volunteers for uniform clothing are arranged to encourage and assist patients to use the WeChat public patient service platform. Through the questionnaire survey, $87.36 \%$ of the respondents paid attention to the service platform of the WeChat public platform in the hospital, indicating that the public platform is a practical platform. The patients for a specific group of people who come to the hospital, based on the promotion in the hospital and the promotion in the hospital, have achieved a certain effect.

Related training to hospital staff, medical staff are the best propagandist, the patient's trust to the medical and nursing staff, can indirectly improve the acceptance of the WeChat public patient service platform. Patients can complete some paying and inquiries through the mobile terminal. Meanwhile, it is also convenient and reduced to the medical staff many interpretations of the work.

Establish guidance, consultation telephone, satisfaction evaluation and other feedback methods, the use of problems may consult the guidance or call consultation, such as comments or suggestions can also be comments through the way. After receiving the feedback of the patient, if it is a personal problem to solve the feedback immediately, the system problems or requirements enter the PDCA demand management process, determine the completion time node and give feedback.

\section{The Highlight of the Construction of the WeChat Public Patient Service Platform}

Source distribution. Since there are advantages and disadvantages of window registration and online registration, the two forms coexist. In order to manage different people fairly and scientifically, two types of registration can not share the same number pool. It is necessary to allocate the number source reasonably. According to the extent of the online registration, the proportion of the online number sources should be adjusted. With about $20 \%$ of the source to the online registered patients, as the platform to achieve a higher level, the amount of use is greatly increased, the general allocation of $50 \%$ of the source to the online registered patients, if the use of the future use of improved, reduce the opening of the window, but also according to specific situation specific analysis, into the adjustment.

Patient binding card. When the patient first uses the WeChat public patient service platform, the patient needs to bing the card operation. The patients who have not been diagnosed, enter the name, ID card number and cell phone number. The system automatically generates a unique identification code for the patient, that is, the outpatient number. After receiving the patient's information, the patient will provide all the outpatient numbers that all patients have been used for patient selection. Because of the reasons left over by history, some patients have more than one outpatient number, some patients have their own common outpatient number, related information of previous visits, rough allocation may cause inconvenience, so that the patient's own choice is also considered respect to the patient, and provide certain benefits.

Refund management. In the protection of hospital finance, it is also the protection of the safety of the patient's property, the patient can not be refunds through mobile phone appointment not to see the day of the visit, which can not be refundable. If it is seen on the day of visit or within three days, the refund of invoices can be refunded through the window, and it can not be refunded through the mobile phone, the invoice refunds must be made up to the window.

\section{The Service Function and Highlights of the WeChat public patient service platform}

The platform mainly provides services through specific interfaces by opening the interface of WeChat public service platform. Its custom menus include up to 3 first level menus, each level 
menu has no more than 5 level two menus. The system administrator can add and delete the first level menu and the second - level menu in the background, and experience the Internet medical treatment mode by the mobile device.

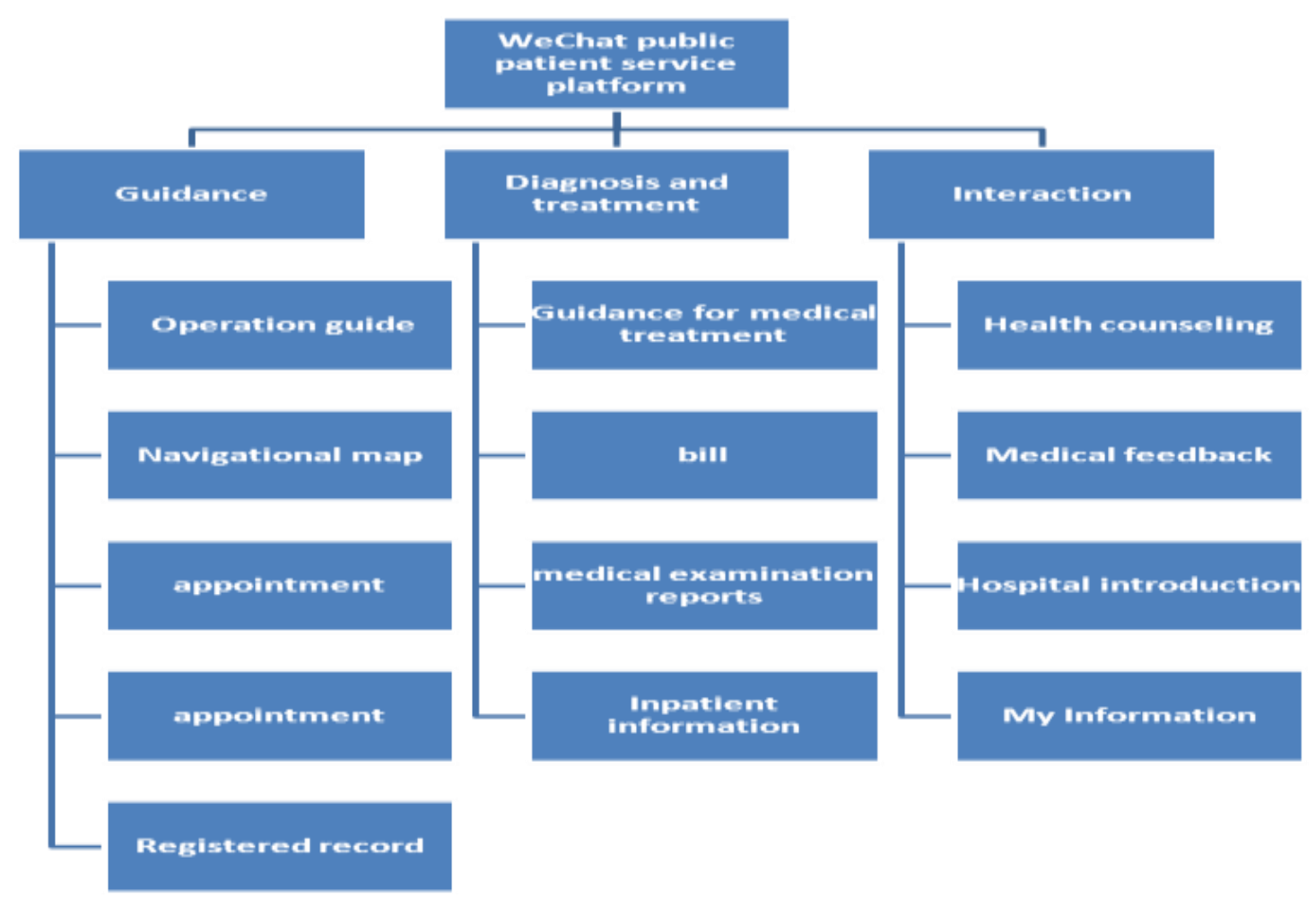

Figure 1. The system architecture

Highlight one: scan code payment in the consultation room. Many patients are registered without the use of online, need to pay the fee to the window, and to return to the window, room, between the terminal department, very troublesome, using a mobile phone card can also get payment information, but the operation is a bit cumbersome, providing direct medical scanning system to provide a two-dimensional code to provide. Then The cost information can be obtained and paid, efficient and convenient, giving patients one click experience.

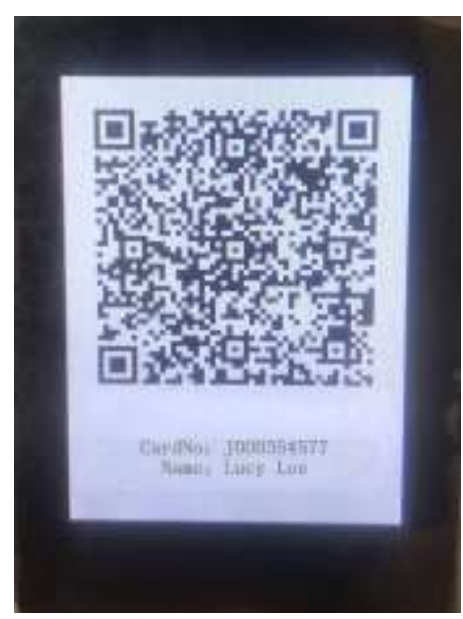

Figure 2. Example diagram

Highlight two: convenient registration for admission to hospital. Due to more revisit patients, most of them belong to radiotherapy and chemotherapy, there is a exclusive doctor. Sometimes the patient will go to the ward to ask the doctor directly if they do not know if they comply with the conditions of hospitalization. When they know that they can return to the hospital after they can be hospitalized, they will go back to the hospital and go back to the ward. In view of this special and universal condition, the doctor can directly handle the registration procedures for the hospital after 
the patient is registered by the mobile terminal directly in the ward, so that the patient's time and strength are greatly saved.

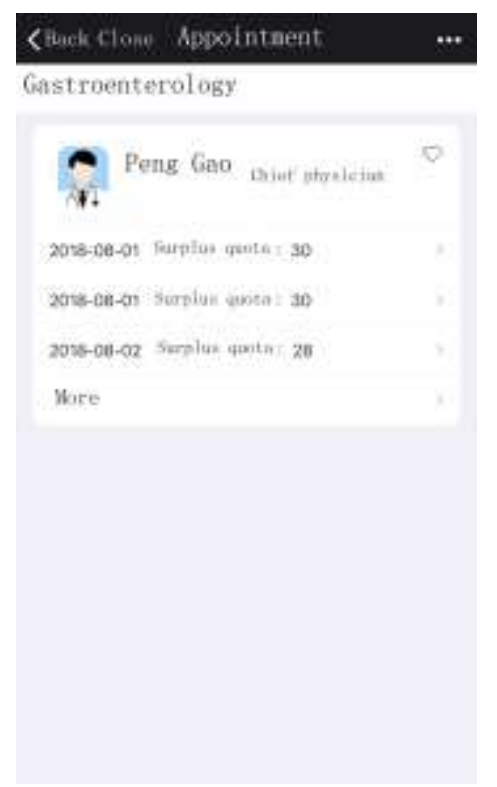

Figure 3. Example diagram

Highlight three: interacting with doctors online. Among the specialties of tumor diseases in specialized hospitals, many patients are revisit patients, and the demand for interaction with the attending doctors is imminent. The system provides a special two-dimensional code for each doctor. The patient is linked to the exclusive home page of the WeChat public patient service platform by scanning the code two-dimensional code. It is not only convenient to see the doctor's outpatient time and register, but also to consult and communicate with the doctor. Professional advice is also convenient for doctors to follow up the discharged patients.

\section{Summary and Prospect}

With the rapid development of Internet and medical treatment, more and more people are willing to accept the mode of medical treatment. From the previous suspicion and conflict to the active and active use, it is also a great challenge to the development of information technology in the hospital. In order to conform to the development of the times, in order to achieve the real convenience of the people, in order to effectively improve the level of medical service, the current level is still far from enough. We should deeply understand the urgent needs of the people, continue to expand the new functions and new applications, and deepen the principle of the convenience of the people. For example, using artificial intelligence, we design Turing robot to answer some simple operation procedures and ask questions frequently. In the case of hospital navigation, the patient can locate the location on the map, search the location of the Department you want to go, and even find the parking space by positioning.

In this age of intelligence, the medical cause which is closely related to the people's livelihood can not be lagged behind. Only by continuous reform and innovation can the hospital grasp the pulse of the times, bring more convenient medical experience for the people and build a harmonious medical atmosphere. 


\section{References}

[1] Sun X M, Wang H J, Communication S O, et al. Survey and Analysis on the Construction and Application Status of Wechat Public Service Platform of Public Library_ Case Study of Provincial Public Libraries in Mainland China[J]. Journal of Library \& Information Sciences in Agriculture, 2016.

[2] Wan Z, Cai J, Cao R. Investigation and analysis of content construction status related to journal articles and contents in We Chat public accounts: Taking RCCSE authoritative academic journals as examples[J]. Chinese Journal of Scientific \& Technical Periodicals, 2017.

[3] Liu Y, Wang X J. Investigation and Analysis on Medical Insurance of College Students in Harbin[J]. Management Observer, 2017.

[4] Peng P, Jin-Dong L V, Chen L, et al. Construction of Hospital Service System Based on WeChat Public Platform[J]. Journal of Medical Informatics, 2015.

[5] Shen Y, Wang J, Yuan H E, et al. Hospital Brand Construction Based on WeChat Public Platform[J]. Hospital Administration Journal of Chinese Peoples Liberation Army, 2017.

[6] Liu Q Q, Xu J, Wang Z Y, et al. Construction and application of the wechat public platform of pharmaceutical care[J]. Pharmaceutical Care \& Research, 2017, 17(4):302-304.

[7] Gao Y. Research on the Application of WeChat Public Platform in College Party Construction[C]// International Conference on Humanities and Social Science Research. 2017.

[8] Zhang C, University J C. Application of Wechat Platform in Party Construction in Colleges and Universities[J]. China Computer \& Communication, 2017.

[9] Liu C, Shi Y. The application of WeChat quick response code in WeChat official accounts[C]// Technology, Networking, Electronic and Automation Control Conference. IEEE, 2018:14-17. 